\title{
Structural analysis on Salmonella effector SseK3-UDP complex
}

\author{
Ivy Yeuk Wah Chung ${ }^{1}$ and Miroslaw Cygler ${ }^{1}$ \\ ${ }^{1}$ Department of Biochemistry, University of Saskatchewan, 107 Wiggins Road, Saskatoon, SK, \\ Canada S7N 5E5.
}

Salmonellae are Gram-negative bacterial pathogens that infect a wide variety of animals. However, the manifestation of the disease varies between hosts. Salmonella enterica is a common food borne human pathogen with $S$. Typhimurium causing gastroenteritis and $S$. Typhi causing typhoid fever. $S$.

Typhimurium uses the type III secretion system (T3SS) to deliver virulent effectors into the host to promote intracellular growth and replication. A group of T3SS effectors known as the Salmonella secreted effector K (SseK 1, 2, and 3) was identified based on their amino acid sequence similarity to the $\mathrm{NleB}$ effector protein present in pathogenic Escherichia coli strains. NleB functions as a glycosyltransferase that transfers a N-Acetylglucosamine moiety to an arginine residue via an Nglycosidic linkage. The known targets are FAS-associated death domain protein (FADD), death domain of TRADD and RIPK1. This modification blocks death receptor signaling and apoptosis.

Here we report the structure of SseK3 in complex with uridine diphosphate (UDP). SseK3 was shown to modify TRADD weakly but not FADD. All members of the NleB/SseK family share a conserved DXD motif that is known to bind $\mathrm{Mn}^{2+}$ and/or coordinate the sugar donor in other GT-A glycosyltransferases. Effector SseK3 shows the classical GT-A glycosyltransferase fold with several $\alpha$-helices flanking a twisted $\beta$-sheet in addition to an $\alpha$-helical insertion. The crystals were grown in the presence of UDP Nacetylglucosamine, a known sugar donor for NleB family, yet clear density is only observed for UDP but not $\mathrm{N}$-acetylglucosamine. Atom $\mathrm{O} 2$ on UDP forms a hydrogen bond with the backbone amide of $\mathrm{Phe}^{53}$, while the N3 atom forms a hydrogen bond with the backbone carbonyl oxygen of Phe ${ }^{53}$. In addition, the uracil ring of UDP forms pi-stacking interaction with $\operatorname{Trp}^{52}$ and Phe ${ }^{190}$. The backbone carbonyl oxygen of $\mathrm{Gln}^{51}$ and hydroxyl group of $\mathrm{Tyr}^{224}$ form hydrogen bonds with the ribose $\mathrm{C} 2$ hydroxyl while the $\mathrm{C} 3$ hydroxyl forms hydrogen bond with the backbone amide of $\mathrm{Ala}^{227}$. Effector SseK3 is the first member of the $\mathrm{NleB} / \mathrm{SseK}$ family to be structurally characterized, information gained from this study can be used for homology modeling to further our understanding on this family of virulent effectors. 